\title{
THE EQUATORIAL NEUTRAL THERMOSPHERIC RESPONSE TO GEOMAGNETIC
}

\author{
A. G. Burns and T. L. Killeen
}

Department of Atmospheric, Oceanic and Space Sciences, University of Michigan.

\begin{abstract}
Thermospheric temperature and neutral density measurements from Dynamics Explorer 2 (DE 2) have been used to study the time-dependent response of the equatorial thermosphere to geomagnetic forcing. Data taken following the onsets of several different isolated geomagnetic storms have been used in a superposed-epoch scheme to investigate the characteristic response of the density and temperature structure of the equatorial thermosphere at $\sim 350 \mathrm{~km}$ altitude in the moming and evening local-time sectors. The principal poststorm-onset features that have been observed are as follows: 1) Two travelling waves, one generated at northern high latitudes and one at southern high latitudes, pass through the equatorial thermosphere, with wave crests crossing the equator at a time lag of 3-6 hours after the storm onset. The maximum neutral density perturbations associated with these waves are $\sim 15$ $25 \%$ for atomic oxygen and $\sim 30-40 \%$ for molecular nitrogen. 2) Following the passage of these travelling waves, the densities and temperatures in the evening sector return relatively rapidly to their undisturbed state, whereas those in the morning sector have significant variations at longer lag times. In particular, significant increases in neutral density and temperature occur in the morning sector about 12 hours after the onset of the storm. The $\mathrm{N}_{2}$ density enhancements are seen earliest in the summer hemisphere, while the $O$ density enhancements are seen earliest in the winter hemisphere.
\end{abstract}

\section{Introduction.}

High-latitude, storm-related changes in neutral composition have generated a great deal of interest since it was first suggested that they could be responsible for the depletions in electron density that occur near the $\mathrm{F}_{2}$ peak. Enhancements of $\mathrm{N}_{2}$ and $\mathrm{O}_{2}$ densities occur at these latitudes during storms, while $O$ densities are depleted at altitudes below about $300 \mathrm{~km}$ and enhanced above this height [e.g., Mayr et al., 1978].

At low latitudes, enhancements in the density of all species are observed during geomagnetic storms [e.g., Prölss 1980; 1982; Mayr et al., 1978], but these increases in density are much smaller than those observed at high latitudes. Because the densities of all species are enhanced, the $\mathrm{N}_{2}$ to $\mathrm{O}$ ratio does not change much along a satellite orbit in response to increased geomagnetic forcing. However, the relatively large changes in veutral density that do occur are important both because they can perturb satellite orbits and because they may act as tracers for changes that occur in other neutral parameters in the oquatorial thermosphere.

Prölss [1982] indicated that 2 main mechanisms may be responsible for these observed changes in equatorial composition. First, material could be transported by largescale circulation [e.g, Volland and Mayr 1971; Mayr and

Copyright 1992 by the American Geophysical Union.

Paper number 92GL00522

$0094-8534 / 92 / 92 \mathrm{GL}-00522 \$ 03.00$
Volland 1974]. Second, travelling ionospheric disturbances could cause changes in composition at low latitudes. Prölss found that each of these mechanisms could explain some of the observed features of equatorial composition changes.

The mechanisms that can cause changes in equatorial temperatures at low latitudes during geomagnetic storms are similar to those that affect composition. Richmond [1979] concluded that circulation is more important energetically than waves, but that the effects of circulation should take 9-12 hours to propagate to the equator, whereas waves can traverse the distance in 4-5 hours. Equatorial heating during geomagnetic storms has also been explained using circulation by a number of other workers [e.g., Volland and Mayr, 1971; Mayr and Volland, 1974; Mayr et al., 1985]. Observational evidence supports these calculations of temperature increases during storms. Equatorial temperature enhancements of the order of $50-200 \mathrm{~K}$ have been seen by Taeusch et al. [1971] and others.

In this paper we attempt to develop a generalized morphology of the equatorial neutral thermospheric response to geomagnetic forcing to provide further observational evidence of the temperature and density changes that occur near the equator during geomagnetic storms. By looking at 2 local time sectors we gain insight into the diumal variations of this response.

The organization of this paper is as follows. The data analysis techniques are described in section 2 . The data from the evening sector are discussed in section 3 and from the morning sector in section 4. Finally the conclusions that can be drawn from this study are considered in section 5 .

Data Analysis.

In this study we need to isolate storm time effects from normal quiet time variations in temperature and density. Therefore, a long period in which storm conditions do not prevail is needed to isolate the storm-time response to high geomagnetic activity in the equatorial regions from the effects of previous storms. We have arbitrarily assigned a cut-off $\mathrm{K}_{\mathrm{p}}$ value of 5 to separate storm conditions from times at which storms are not occurring. Thus, to isolate individual storms we have required a sustained period of 24 hours preceding the onset of the geomagnetic storm in which $\mathrm{K}_{\mathrm{p}}$ values are always smaller than this value. In practice this criterion works well, with $\mathrm{K}_{\mathrm{p}}$ typically being much smaller than 5 in the period before the onset of the storm in most of the storms considered (see Figure 1 for the agglomeration of the $K_{p}$ values that occurred in the storms used in this study). After the storm commencement the values of $\mathrm{K}_{\mathrm{p}}$ vary greatly. Given this variation it is surprising that any coherency occurs in our results. However, as such coherency does occur, it suggests that the changes that are forced in the low latitude thermosphere by geomagnetic storms are fundamental, and that they depend on the occurrence of a storm, rather than the specific morphology of the storm. 


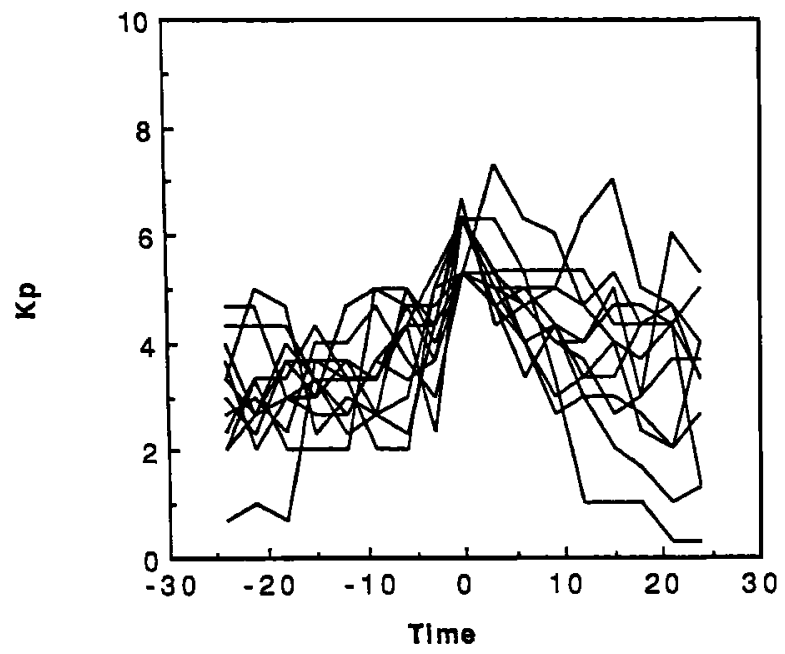

Figure 1. This is an agglomeration of the values of $\mathrm{K}_{\mathrm{p}}$ used in this study. The bottom axis represents the time in hours before and after the onset of the storm. Negative values represent time before the onset of the storm.

We looked at density and temperature data from the DE2 satellite during storms which met the above requirements in the northern hemisphere winter months (November, December and January) of 1981/82 and 1982/83 and found a number of cases in which these data existed. The storms were separated into 2 different local time sectors, one comprising of the evening hours (18-20 hours local solar time) and one comprising of the moming hours (4-6 hours local solar time). Typically, there were insufficient equatorial data available to produce any meaningful results from outside these two local time periods during the 2 winters studied. This study is restricted to the equatorial region because the eccentricity of the satellite orbit precludes the gathering of composition data through much of the orbit. We binned the data in 3 hour bins by time after the onset of the geomagnetic disturbance and by $5^{\circ}$ bins in latitude from -30 to $+30^{\circ}$. $\mathrm{K}_{\mathrm{p}}$ was used as an indicator of geomagnetic activity primarily because of its widespread usage. The problems of resolution that are associated with this index [Prölss, 1982] are noted, but, given the time resolution of 3 hours that we use, an index with similar resolution is justified. When $O$ densities were considered we found that 9 storms in the evening sector met the criteria listed above, while 4 storms met these criteria in the morning sector.

The specific data sets had to be further reduced to separate storm-time phenomena from normal, quiet-time temporal variations. The temperature data were all assumed to represent exospheric temperatures as the perigee of the satellite did not fall much below $300 \mathrm{~km}$ during the period of the study, so no further processing was undertaken on these data. Meaningful insight into density variations could only be gained by reducing the density data to a constant height or pressure surface. In this case the density data were reduced to a constant height surface of $350 \mathrm{~km}$ by assuming diffusive equilibrium using the temporally equivalent temperature data. The errors associated with this assumption of diffusive equilibrium were minimized by choosing a height within the altitude range of the data used, rather than the more usual 300 $\mathrm{km}$.
A control data set was also calculated after a similar 2 hour relaxation period for times in which no storm occurral Storm time enhancements and depletions of the data were calculated by subtracting the control data from the storm $t^{-}$ data and then dividing by the control data. The resulting $d^{2}$ set represents the fractional change of storm time data from control data and is represented this way in the remainis figures. Percentage changes can be obtained by multiply
these fractional changes by 100 .

\section{Storm Time Effects in the Evening Sector}

The density changes that occur at $350 \mathrm{~km}$ in the everisector ( $18-20$ hours local solar time) as a result of the stat are given Figure 2. The most noticeable feature in the density plots is the large enhancements of density that are sem 3 to 6 hours after the onset of the storm. These enhancemerts are followed by density depletions 6-12 hours after the onset of the storm for $\mathrm{N}_{2}$ and 6-9 hours after the onset of the stom for $\mathrm{O}$. The magnitude of the enhancements is $30-40 \%$ for $\mathrm{M}_{2}$ densities and $15-25 \%$ for $O$ densities. Because of the oscillatory nature of these changes we ascribe them to transverse wave activity. The density changes described above are equivalent to a vertical wave amplitude of $8-10 \mathrm{~km}$ : the difference in the density amplitudes of the 2 species occus because the scale height of $O$ is $60-80 \mathrm{~km}$, while the scale height of $\mathrm{N}_{2}$ is about $30 \mathrm{~km}$; thus a wave passing through the thermosphere causes a proportionally smaller change in 0 densities than in $\mathrm{N}_{2}$ densities. In general, these densities have returned to their quiet time values some 12 hours after the onset of the storm.

Temperature changes in storm time relative to quiet tim also show a period of enhancement followed by a period of depletion (Figure 3). The initial period of temperature enhancement occurs somewhat before the density increases This time difference is disguised by the coarse time resolutice used in our study, but the difference is clear at the higher latitudes (near 30 degrees latitude in both hemispheres). Tis:

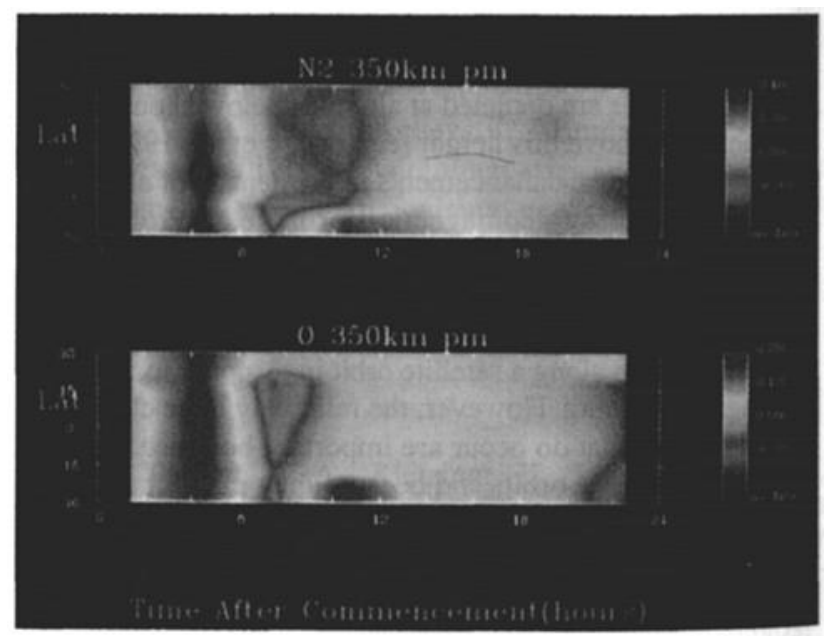

Figure 2. This figure shows $\mathrm{N}_{2}$ (top) and $\mathrm{O}$ (bottom) density changes that result from a storm in the evening local time sector. The vertical axis is the geographic latitude, with the summer hemisphere on the bottom, while the horizontal axis is the time after the onset of the storm. The color scale is given in the fractional change between storm time and the control case 


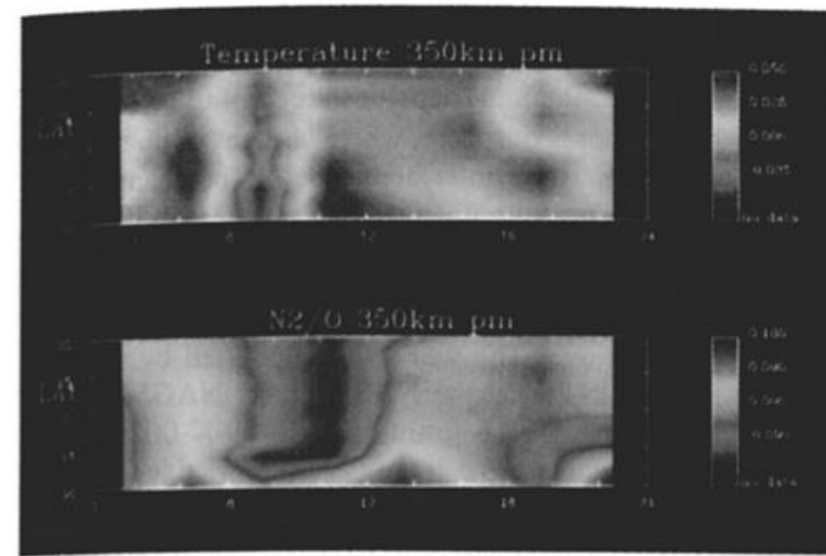

Figure 3. This is the same as Figure 2, but for temperature (top) and $\mathrm{N}_{2} / \mathrm{O}$ ratio (bottom).

change is consistent with a wave interpretation for these data; the initial phase of this wave is comprised of downward winds which cause compressional heating. The corresponding temperature increase of about $5 \%$ is equivalent to the wave amplitude calculated from the density changes, as the compressional heating associated with a wave which has an amplitude of $10 \mathrm{~km}$ is about $70 \mathrm{~K}$ in 3 hours or about $6 \%$ at $1200 \mathrm{~K}(1200 \mathrm{~K}$ is roughly the average quiet-time temperature in the evening sector near the equator during solar maximum).

In the next phase of the wave upward winds cause adiabatic cooling and density increases on a constant height surface. The total change in temperature that results from this cooling reduces the temperature below its normal quiet-time value as is seen in Figure 3. Further support for the assertion that these temperature changes are a result of wave activity is gained when the phase differences in the temperatures are considered. Temperature increases are seen at $30^{\circ}$ latitude in both hemispheres in the time bin prior to the bin in which they are seen at the equator. This latitudinal variation also indicates that waves are generated in both hemispheres at about the same time during a geomagnetic storm, so the two waves meet near the equator. This interpretation also explains the observation of larger density increases near the equator [Prölss, 1982], where the 2 waves will constructively interfere, than at $30^{\circ}$ latitude. The later enhancements in temperature that are seen in Figure 3 are not so readily explainable and will be left for a later study.

As both $\mathrm{N}_{2}$ densities and $\mathrm{O}$ densities are increasing and decreasing in the same bins the ratio of $\mathrm{N}_{2}$ to $\mathrm{O}$ does not change very much. Changes in this ratio are typically of the order of $9 \%$ as a result of the wave activity that is discussed sbove.

\section{Storm Effects in the Morning Sector.}

The morphology of the changes in density that occur in the moming sector (4-6 hours local solar time - Figure 4) is very different from that of the changes that occur in the evening sector. The $O$ density variations still show evidence of wave activity some $3-6$ hours after the onset of the storm. The amplitude of the increase in $O$ density associated with this wave is about $30-40 \%$, a figure that is somewhat larger than the enhancements in $O$ density in the evening sector. There are also changes in $\mathrm{N}_{2}$ density associated with this wave, but these changes are hidden by the need to choose a scale that

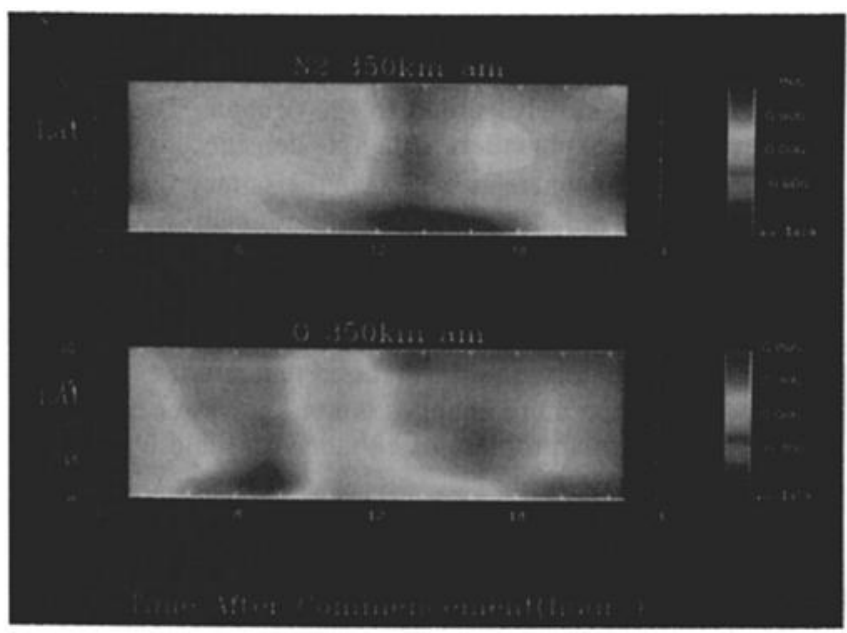

Figure 4. This is the same as figure 2, but in the morning sector.

included the later changes in $\mathrm{N}_{2}$ density. Neutral density variations are dominated by these later changes that occur some 12 hours after the onset of the storm. Values of $\mathrm{N}_{2}$ density in storm time are as much as $180 \%$ greater than the equivalent quiet time densities in the summer hemisphere near $30^{\circ}$ latitude 12 hours after the onset of the storm and as much as $90 \%$ elsewhere. The later enhancements of $\mathrm{N}_{2}$ density are first seen $9-12$ hours after the beginning of the storm in the summer hemisphere and persist for at least 12 hours after this time. $O$ densities also increase within a similar time frame. However, these enhancements in $\mathrm{O}$ density are smaller than those of $\mathrm{N}_{2}$ and are first seen in the winter hemisphere rather than in the summer hemisphere. The later $\mathrm{N}_{2}$ density increases are much larger than those associated with the initial wave activity and must be generated by a different process.

At geomagnetically quiet times $\mathrm{N}_{2}$ densities are considerably larger in the summer hemisphere than in the winter hemisphere, while $O$ densities are slightly larger in the winter hemisphere than in the summer hemisphere [Mayr et al., 1978]. A simple change in circulation that involves stronger equatorward winds in both hemispheres would result in enhanced $\mathrm{N}_{2}$ densities being seen at the higher latitudes in

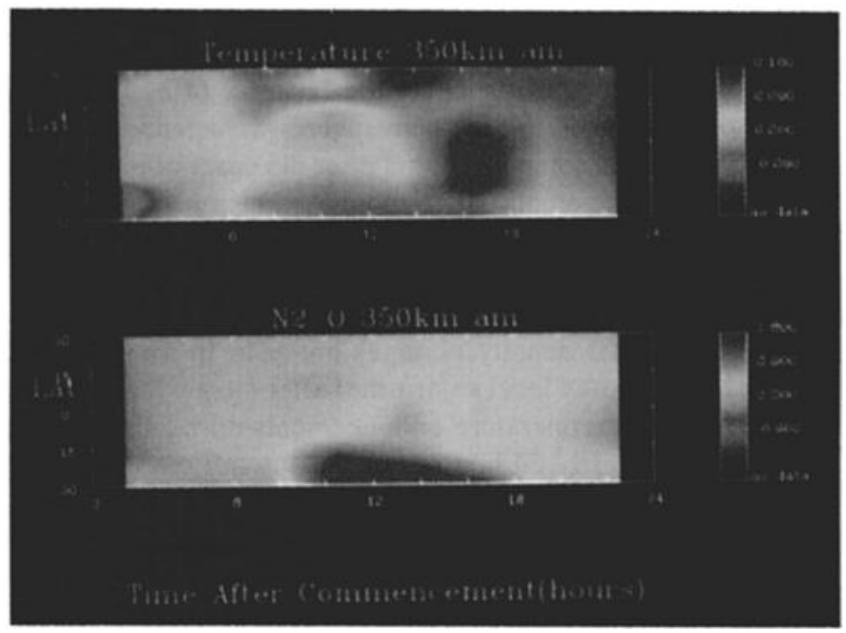

Figure 5. This is the same as Figure 3, but in the morning sector. 
the summer hemisphere first, while smaller enhancements in $O$ density would be seen in the higher latitudes of the winter hemisphere and slowly spread to the equator. The problem with this simple explanation is that $\mathrm{N}_{2}$ densities are enhanced in the winter hemisphere as well as in the summer hemisphere, and $O$ densities are also enhanced in both hemispheres. This problem is further exacerbated as the direct transportation of material from the auroral regions into this local time sector is unlikely because of the effects of corotation [Burns et al., 1991]. Unfortunately, the mechanism that could cause these density and temperature changes is not known for certain, so the solution to this problem of enhanced $\mathrm{N}_{2}$ and $\mathrm{O}$ densities requires further study.

The temperature enhancements that are seen some 12 hours after the onset of the storm (Figure 5) may be caused by increased compressional heating as the greater convergence of meridional winds that occurs during storm time in this region results in stronger downward winds, completing the circulation cell set up by heating in the auroral zone at the time of geomagnetic storms. An alternative explanation could involve the direct advection of heat into the equatorial region by enhanced horizontal winds. Again this problem requires further study before an acceptable solution can be obtained. Whatever the cause of this heating, we find that the temperatures are enhanced by about $10 \%(90-100 \mathrm{~K})$ some 12 hours after the onset of the storm in this local time sector. These temperature increases are first seen at higher latitudes and are later seen near the equator.

The $\mathrm{N}_{2} / \mathrm{O}$ values respond very strongly to the storm $(\sim 180$ $\%$ ) in the summer hemisphere 12 hours after the onset of the storm. Changes in this ratio are minor elsewhere. Thus, the $\mathrm{N}_{2} / \mathrm{O}$ ratio is not a good tracer for changes that occur at a constant altitude in the equatorial region during geomagnetic storms.

\section{Conclusions.}

We have used Dynamics Explorer 2 low latitude density and temperature measurements to study the generalized time dependent response of the equatorial thermosphere to geomagnetic storms. We find the following:

1) The morphology of the temperature and density changes is very different in the 2 local time sectors used (1820 hours local solar time and $4-6$ hours locil solar time).

2) There is good evidence for the existence of 2 large scale waves, one generated in each hemisphere, propagating into the equatorial regions some 3-6 hours after the onset of the storm. The corresponding amplitude is about $5 \%$ in temperature in the evening sector, with downward winds probably occurring in the initial phase. These waves are seen in both local time sectors.

3) No further density changes are seen in the evening sector (18 - 20 hours local solar time) after the passage of this wave. However temperature enhancements do persist in this sector until some $18-24$ hours after the onset of the storm.
4) Large changes in both $\mathrm{N}_{2}$ and $\mathrm{O}$ density occur about 12 hours after the onset of the storm in the morning sector (4 - 6 hours local solar time). $\mathrm{N}_{2}$ densities are seen to be enhanced first in the summer hemisphere, where changes of up to $180 \%$ occur, and later in the winter hemisphere. The changes in 0 density are smaller, with changes of the order of $30-40 \%$ typically being seen, but they are seen first in the winter hemisphere and later in the summer hemisphere.

Acknowledgements. The work was supported by the NSF grant ATM-8918476, by the NASA grants NAG5-465 and NAGW-1535 and USAF grant F19628-89-K-0026 to the University of Michigan. We gratefully acknowledge the use of WATS data from Mr. N. Spencer. Discussions with Drs. A. E. Hedin, F. G. McCormac and R. G. Roble are gratefully acknowledged.

\section{References.}

Burns, A. G., T. L. Killeen and R. G. Roble, A theoretical study of thermospheric composition perturbations during an impulsive geomagnetic storm, J. Geophys. Res. 26 14153-14167, 1991.

Mayr, H. G. and H. Volland, Magnetic storm dynamics of the thermosphere, I. Atmos. Terr. Phys. 36, 2025-2036, 1974.

Mayr, H. G., I. Harris and N. W. Spencer, Some properties of upper atmosphere dynamics, Rev. Geophvs. Space Phys., 16, 539-565, 1978.

Mayr, H. G., I. Harris, F. Varosi, F. A. Herrero, H. Volland, N. W. Spencer, A. E. Hedin, R. E. Hartle, H. A. Taylor, Jr., L. E. Wharton and G. R. Carignan, On the structure and dynamics of the thermosphere, Adv. Space Res., 5, 283-288, 1985.

Prölss, G. W., Magnetic storm associated perturbation of the upper atmosphere: recent results obtained by satellite-borne gas analyzers, Rev. Geophys. Space Phys. 18, 183-202, 1980.

Prölss, G. W., Perturbation of the low-latitude upper atmosphere during magnetic substorm activity, $L$ Geophys. Res., 87, 526-5266, 1982.

Richmond, A. D., Thermospheric heating in a magnetic storm: dynamic transport of energy from high to low latitudes, $L$ Geophys. Res., 84, 5259-5266, 1979.

Taeusch, D. R., G. R. Carignan and C. A. Reber, Neutral composition variation above $400 \mathrm{~km}$ during a magnetic storm, J. Geophys, Res. 76, 8318-8325, 1971.

Volland, H. and H. G. Mayr, Response of the thermospheric density to auroral heating during geomagnetic disturbances, J. Geophys. Res., 76, 3764 - 3776, 1971.

$\overline{\text { A.G. Burns and T. L. Killeen, Space Physics Research }}$ Laboratory, Department of Atmospheric, Oceanic and Space Sciences, University of Michigan, Ann Arbor, 48109-2143.

(Received: January 13, 1992; accepted: February 21, 1992) 